\title{
Article \\ Constraints on Lorentz Invariance Violation with Multiwavelength Polarized Astrophysical Sources
}

\author{
Qi-Qi Zhou ${ }^{1}\left(\mathbb{D}\right.$, Shuang-Xi $\mathrm{Yi}^{1, * \mathbb{C}}$ and Jun-Jie Wei ${ }^{2,3, * \mathbb{C}}$ and Xue-Feng $\mathrm{Wu}^{2,3, *(\mathbb{C})}$ \\ 1 School of Physics and Physical Engineering, Qufu Normal University, Qufu 273165, China; \\ zhouqiqi0107@sina.com \\ 2 Purple Mountain Observatory, Chinese Academy of Sciences, Nanjing 210023, China \\ 3 School of Astronomy and Space Sciences, University of Science and Technology of China, Hefei 230026, China \\ * Correspondence: yisx2015@qfnu.edu.cn (S.-X.Y.); jjwei@pmo.ac.cn (J.-J.W.); xfwu@pmo.ac.cn (X.-F.W.)
}

check for updates

Citation: Zhou, Q.-Q.; Yi, S.-X.; Wei, J.-J.; Wu, X.-F. Constraints on Lorentz Invariance Violation with Multiwavelength Polarized Astrophysical Sources. Galaxies 2021, 9, 44. https://doi.org/10.3390/ galaxies 9020044

Academic Editor: Marco Schreck

Received: 14 April 2021

Accepted: 16 June 2021

Published: 20 June 2021

Publisher's Note: MDPI stays neutral with regard to jurisdictional claims in published maps and institutional affiliations.

Copyright: (c) 2021 by the authors. Licensee MDPI, Basel, Switzerland. This article is an open access article distributed under the terms and conditions of the Creative Commons Attribution (CC BY) license (https:// creativecommons.org/licenses/by/ $4.0 /)$.

\begin{abstract}
Possible violations of Lorentz invariance (LIV) can produce vacuum birefringence, which results in a frequency-dependent rotation of the polarization plane of linearly polarized light from distant sources. In this paper, we try to search for a frequency-dependent change of the linear polarization angle arising from vacuum birefringence in the spectropolarimetric data of astrophysical sources. We collect five blazars with multiwavelength polarization measurements in different optical bands $(U B V R I)$. Taking into account the observed polarization angle contributions from both the intrinsic polarization angle and the rotation angle induced by LIV, and assuming that the intrinsic polarization angle is an unknown constant, we obtain new constraints on LIV by directly fitting the multiwavelength polarimetric data of the five blazars. Here, we show that the birefringence parameter $\eta$ quantifying the broken degree of Lorentz invariance is limited to be in the range of $-9.63 \times 10^{-8}<\eta<6.55 \times 10^{-6}$ at the $2 \sigma$ confidence level, which is as good as or represents one order of magnitude improvement over the results previously obtained from ultraviolet/optical polarization observations. Much stronger limits can be obtained by future multiwavelength observations in the gamma-ray energy band.
\end{abstract}

Keywords: astroparticle physics; gravitation; polarization; multiwavelength

\section{Introduction}

Lorentz invariance, which says that the relevant physical laws of a non-accelerated physical system remain invariant under Lorentz transformations, is a fundamental symmetry of Einstein's theory of relativity. However, many quantum gravity theories seeking to unify quantum mechanics and general relativity predict that Lorentz invariance may be broken at the Planck energy scale $E_{\mathrm{pl}} \approx 1.22 \times 10^{19} \mathrm{GeV}$ [1-8]. Although these high energies are unattainable experimentally, tiny deviations from Lorentz invariance may still exist at lower energies, motivating sensitive tests of Lorentz invariance.

In the photon sector, vacuum dispersion and vacuum birefringence are both significant characteristics of Lorentz invariance violation (LIV). Although these effects are believed to be very tiny at energies $\ll E_{\mathrm{pl}}$, they can become detectable by accumulating over large distances. Vacuum dispersion causes arrival-time differences of photons with different energies emitted simultaneously from an astrophysical source. Many attempts to set constraints on LIV have been performed based on time-of-flight tests of high energy photons [8-28]. In some specific cases, especially in loop quantum gravity, the deviations from Lorentz invariance take a particular form in which photons with right- and lefthanded polarization propagate at different velocities. This leads to an energy-dependent rotation of the polarization vector of the linearly polarized photons, known as vacuum birefringence. Hence, Lorentz invariance can also be tested with astrophysical polarization measurements [4,29-48]. As polarization measurements are more sensitive than time-offlight measurements by a factor $\propto 1 / E$, where $E$ is the energy of the light, more stringent 
constraints on LIV result from the former rather than the latter [21]. However, some Lorentzviolating theories do not predict any vacuum birefringence, so limits from time-of-flight measurements are most interesting for investigating nonbirefringent effects.

In the literature, the linear polarization measurements have been applied to set limits on the birefringence parameter $\eta$. The presence of linear polarization in the $\gamma$-ray band of gamma-ray bursts (GRBs) provides the current best limits on $\eta$, i.e., $\eta<\mathcal{O}\left(10^{-16}\right)$ [29-33]. These upper limits are obtained based on the indirect argument that vacuum birefringence would significantly deplete the net polarization of the signal over a broad bandwidth. In addition, Fan et al. [39] used multiwavelength polarimetric data in order to directly search for a frequency-dependent change of the linear polarization angle resulting from vacuum birefringence. They obtained a combined limit of $-2 \times 10^{-7}<\eta<1.4 \times 10^{-7}$ by fitting the multiwavelength polarization observations of the optical afterglows of GRB 020813 and GRB 021004. However, it should be noted that the number of available linear polarization measurements in the $\gamma$-ray band for this particular test is very limited. Therefore, the outcomes of LIV tests lack significant statistical robustness even though some upper limits of $\eta$ are extraordinarily small. It is fortunate that some astrophysical sources with multiwavelength polarization measurements in the optical band have been detected. Using different multiwavelength polarimetric data to constrain LIV is always helpful.

In this work, we gather 37 groups of multiwavelength polarization observations from five blazars to test LIV through the method that was discussed in Fan et al. [39]. The contents of this paper are organized as follows. In Section 2, we describe the method of constraining LIV. The multiwavelength polarization samples at our disposal and the resulting constraints on LIV are presented in Section 3. The physical implications of our results are discussed in Section 4. Finally, our conclusions are presented in Section 5.

\section{Method of Constraining LIV}

Considering Lorentz-violating effects, photons with right- and left-circular polarizations will traverse with different group velocities, which can be described using the leading term of a Taylor series expansion in the form [4]

$$
v_{ \pm}=c\left[1 \pm \eta\left(\frac{\hbar \omega}{E_{\mathrm{pl}}}\right)^{n}\right],
$$

where $E_{\mathrm{pl}} \approx 1.22 \times 10^{19} \mathrm{GeV}$ is the Planck energy, the $n$ th-order expansion of the leading term stands for linear $(n=1)$ or quadratic $(n=2)$ energy dependence, \pm represents the different polarization states for photons, and $\eta$ is a dimensionless constant characterizing the degree of the LIV effect. Here, we consider the linearly polarized light from some astronomical sources, which is a superposition of two monochromatic waves with opposite circular polarizations. If Lorentz invariance is broken (i.e., when $\eta \neq 0$ ) then photons with right- and left-handed circular polarizations that are emitted simultaneously from the same source should have different group velocities, leading to a rotation of the polarization vector of a linearly polarized plane. The rotation angle in the differential propagation distance $d L_{(z)}$ can be expressed as [4,47-49]

$$
\mathrm{d} \phi=\eta\left(\frac{\omega l_{p}}{c}\right)^{n+1} \frac{\mathrm{d} L_{(z)}}{l_{p}}
$$

where $l_{p}=\sqrt{\hbar G / c^{3}}=\hbar c / E_{\mathrm{pl}}$ is the Planck length scale. Taking into account the cosmological expansion, the rotation angle $\Delta \phi_{\text {LIV }}$ during the propagation from the source at redshift $z$ to the observer is given by

$$
\Delta \phi_{\mathrm{LIV}}=\frac{\eta}{H_{0}}\left(\frac{l_{p}}{c}\right)^{n}(2 \pi v)^{n+1} F(z, n),
$$


where $v$ is the frequency of the observed light and $H_{0}$ is the Hubble constant. The function $F(z, n)$ depends on the cosmology model. For a flat $\Lambda$ CDM model,

$$
F(z, n)=\int_{0}^{z} \frac{\left(1+z^{\prime}\right)^{n} d z^{\prime}}{\sqrt{\Omega_{M}\left(1+z^{\prime}\right)^{3}+\Omega_{\Lambda}}},
$$

where $\Omega_{M}$ and $\Omega_{\Lambda}$ are the cosmological parameters. Since the polarization data used in this work are not sensitive to higher-order terms, here we only consider the linear $(n=1)$ LIV case [39]. We thus have [33,37]

$$
\Delta \phi_{\mathrm{LIV}}(E)=\eta \frac{E^{2}}{\hbar E_{\mathrm{pl}} H_{0}} \int_{0}^{z} \frac{\left(1+z^{\prime}\right) d z^{\prime}}{\sqrt{\Omega_{M}\left(1+z^{\prime}\right)^{3}+\Omega_{\Lambda}}} .
$$

Throughout this paper, we adopt the cosmological parameters $H_{0}=67.36 \mathrm{~km} \mathrm{~s}^{-1} \mathrm{Mpc}^{-1}$, $\Omega_{M}=0.315$, and $\Omega_{\Lambda}=1-\Omega_{M}=0.685$ [50].

When considering both the intrinsic polarization angle $\phi_{0}$ and the rotation angle $\Delta \phi_{\text {LIV }}$ caused by LIV simultaneously, the observed polarization angle at a certain energy $E$ emitted from an astronomical event should consist of two terms

$$
\phi_{\mathrm{obs}}(E)=\phi_{0}+B E^{2},
$$

where the slope $B=\eta \frac{F(z)}{\hbar E_{\mathrm{pl}} H_{0}}$ denotes the contribution from LIV. However, since the emission mechanism of astronomical sources is still poorly understood, it is difficult to distinguish an intrinsic polarization angle at the source from a rotation angle of the polarization plane induced by LIV. Following Fan et al. [39], we simply assume that all photons in the observed bandpass are radiated with the same (unknown) intrinsic polarization angle. This assumption is vulnerable to unknown systematic uncertainties associated with the unknown intrinsic properties of any given sources. In principle, these unknown intrinsic systematic uncertainties can be minimized by analyzing the sizeable multiwavelength polarization data of a given source. However, there are no abundant spectropolarimetry data in the current observations. We explore here the implications and limits that can be set under the simplest assumption that the intrinsic polarization angle is an unknown constant. In this case, if the corresponding $E^{2}$ is taken as an independent variable, the free parameters $\phi_{0}$ and $B$ can be optimized from the linear fitting of the linear polarization measurements in several bands from a given astronomical event. With the fitting results, we then derive the limited range of the birefringence parameter $\eta$. All the results are obtained at the $2 \sigma$ confidence level.

\section{Tests of LIV with Polarized Sources}

It is obvious from Equation (5) that the higher the energy band of the polarization observation and the larger the distance from the source, the better is the constraint on the birefringence parameter $\eta$. However, there are no multiwavelength polarization measurements in the $\gamma$-ray or X-ray energy band, yet. Fan et al. [39] explored the limits of $\eta$ by observations of multiwavelength polarization from the optical afterglows of GRB 020813 and GRB 021004 (see also Ref. [46]). Here, we continue to search for astrophysical sources with multiwavelength polarimetric data that are suitable for testing LIV effects. The optical spectropolarimetry from several well-known blazars have been reported. We collect the high-quality polarimetry data of blazars 3C 66A, S5 0716+714, OJ 287, MK 421, and PKS 2155-304 from Refs. [51-53]. We then apply these polarized blazars to constrain possible birefringence effects in this work.

The linear polarization observations of these five blazars are presented in Table 1, including the following information for each object: the energy bands in which polarization is observed; the observed linear polarization angles; and the corresponding uncertainties of the polarization angles. These polarization observations have been obtained simultaneously 
in five optical bands $(U B V R I)$ for all blazars. The redshifts of the five blazars 3C 66A, S5 0716+714, OJ 287, MK 421, and PKS 2155-304 are 0.444, 0.310, 0.306, 0.031, and 0.116, respectively. Since the optical $(U B V R I)$ polarization measurements are carried out at different time periods, there are several groups of spectropolarimetry data for each blazar. A total of 37 groups of multiwavelength polarimetric data of the five blazars are listed in Table 1. These enable us to carefully investigate the long- and short-term polarization variability of each blazar and to search for LIV effects.

Table 1. Measurements of multiwavelength polarization for different blazars.

\begin{tabular}{|c|c|c|c|c|c|c|c|}
\hline Group & Filter & Polarization Angle $\left({ }^{\circ}\right)$ & $\sigma$ & Group & Filter & Polarization Angle $\left({ }^{\circ}\right)$ & $\sigma$ \\
\hline & & $3 \mathrm{C} 66 \mathrm{~A}$ & & & & OJ 287 & \\
\hline \multirow[t]{5}{*}{1} & $\mathrm{U}$ & 40.5 & 0.7 & 1 & $\mathrm{U}$ & 108.6 & 1.8 \\
\hline & B & 43.1 & 0.6 & & B & 108.1 & 2.5 \\
\hline & $\mathrm{V}$ & 40.1 & 0.6 & & $\mathrm{~V}$ & 108.7 & 1.1 \\
\hline & $\mathrm{R}$ & 35.1 & 0.5 & & $\mathrm{R}$ & 103.7 & 1.6 \\
\hline & I & 32.2 & 1.2 & & I & 98.4 & 3.3 \\
\hline \multirow[t]{5}{*}{2} & $\mathrm{U}$ & 41.0 & 0.6 & 2 & $\mathrm{U}$ & 103.9 & 1.0 \\
\hline & B & 42.0 & 0.6 & & B & 109.3 & 1.5 \\
\hline & $\mathrm{V}$ & 41.0 & 0.6 & & $\mathrm{~V}$ & 105.2 & 1.5 \\
\hline & $\mathrm{R}$ & 35.3 & 0.5 & & $\mathrm{R}$ & 101.5 & 1.0 \\
\hline & I & 31.9 & 1.2 & & I & 106.2 & 4.2 \\
\hline \multirow[t]{5}{*}{3} & $\mathrm{U}$ & 39.3 & 0.5 & 3 & $\mathrm{U}$ & 105.2 & 1.3 \\
\hline & B & 40.2 & 0.8 & & B & 106.4 & 1.7 \\
\hline & $\mathrm{V}$ & 38.1 & 0.8 & & $\mathrm{~V}$ & 106.3 & 1.6 \\
\hline & $\mathrm{R}$ & 33.8 & 0.4 & & $\mathrm{R}$ & 101.4 & 1.2 \\
\hline & I & 35.3 & 1.7 & & I & 97.6 & 1.9 \\
\hline \multirow[t]{5}{*}{4} & $\mathrm{U}$ & 39.6 & 1.2 & 4 & $\mathrm{U}$ & 106.8 & 1.5 \\
\hline & B & 41.6 & 1.3 & & B & 107.8 & 1.4 \\
\hline & $\mathrm{V}$ & 39.7 & 1.4 & & $\mathrm{~V}$ & 103.5 & 1.4 \\
\hline & $\mathrm{R}$ & 33.8 & 1.7 & & $\mathrm{R}$ & 103.2 & 0.9 \\
\hline & I & 36.0 & 3.1 & & I & 104.5 & 3.2 \\
\hline \multirow[t]{5}{*}{5} & $\mathrm{U}$ & 51.3 & 0.2 & 5 & $\mathrm{U}$ & 105.7 & 1.3 \\
\hline & B & 51.7 & 0.2 & & B & 107.4 & 2.1 \\
\hline & $\mathrm{V}$ & 50.4 & 0.2 & & $\mathrm{~V}$ & 102.1 & 1.4 \\
\hline & $\mathrm{R}$ & 48.1 & 0.2 & & $\mathrm{R}$ & 102.3 & 0.8 \\
\hline & I & 45.7 & 0.5 & & I & 96.8 & 3.2 \\
\hline \multirow[t]{5}{*}{6} & $\mathrm{U}$ & 39.3 & 0.2 & 6 & $\mathrm{U}$ & 105.6 & 1.3 \\
\hline & B & 40.2 & 0.3 & & B & 108.5 & 1.4 \\
\hline & $\mathrm{V}$ & 39.4 & 0.3 & & $\mathrm{~V}$ & 103.6 & 1.2 \\
\hline & $\mathrm{R}$ & 38.3 & 0.2 & & $\mathrm{R}$ & 102.0 & 1.3 \\
\hline & I & 36.9 & 0.6 & & I & 102.6 & 2.9 \\
\hline \multirow[t]{5}{*}{7} & $\mathrm{U}$ & 38.2 & 0.2 & 7 & $\mathrm{U}$ & 108.2 & 1.8 \\
\hline & $\mathrm{B}$ & 39.1 & 0.2 & & B & 109.8 & 2.8 \\
\hline & $\mathrm{V}$ & 38.2 & 0.3 & & $\mathrm{~V}$ & 104.0 & 2.1 \\
\hline & $\mathrm{R}$ & 37.2 & 0.2 & & $\mathrm{R}$ & 101.0 & 1.7 \\
\hline & $\mathrm{I}$ & 35.7 & 0.4 & & I & 99.8 & 3.1 \\
\hline \multirow[t]{6}{*}{8} & $\mathrm{U}$ & 35.0 & 0.4 & 8 & $\mathrm{U}$ & 107.5 & 1.3 \\
\hline & B & 34.8 & 0.3 & & B & 108.7 & 1.7 \\
\hline & $\mathrm{V}$ & 34.5 & 0.4 & & $\mathrm{~V}$ & 104.2 & 1.4 \\
\hline & $\mathrm{R}$ & 32.6 & 0.3 & & $\mathrm{R}$ & 100.9 & 1.0 \\
\hline & $\mathrm{I}$ & 31.8 & 0.6 & & I & 94.0 & 3.2 \\
\hline & & S5 $0716+714$ & & 9 & $\mathrm{U}$ & 54.6 & 1.1 \\
\hline \multirow[t]{5}{*}{1} & $\mathrm{U}$ & 22.5 & 0.4 & & B & 53.4 & 1.2 \\
\hline & B & 23.5 & 0.3 & & V & 51.7 & 1.6 \\
\hline & $\mathrm{V}$ & 23.3 & 0.3 & & $\mathrm{R}$ & 44.1 & 1.1 \\
\hline & $\mathrm{R}$ & 23.2 & 0.3 & & I & 44.8 & 2.8 \\
\hline & I & 22.6 & 0.5 & 10 & $\mathrm{U}$ & 65.5 & 0.7 \\
\hline \multirow[t]{5}{*}{2} & $\mathrm{U}$ & 10.8 & 0.2 & & B & 66.9 & 0.7 \\
\hline & B & 12.1 & 0.3 & & $\mathrm{~V}$ & 65.3 & 0.7 \\
\hline & $\mathrm{V}$ & 13.5 & 0.3 & & $\mathrm{R}$ & 64.4 & 0.5 \\
\hline & $\mathrm{R}$ & 12.6 & 0.3 & & I & 63.6 & 1.4 \\
\hline & I & 12.8 & 0.5 & 11 & $\mathrm{U}$ & 68.8 & 0.3 \\
\hline
\end{tabular}


Table 1. Cont.

\begin{tabular}{|c|c|c|c|c|c|c|c|}
\hline Group & Filter & Polarization angle $\left({ }^{\circ}\right)$ & $\sigma$ & Group & Filter & Polarization angle $\left({ }^{\circ}\right)$ & $\sigma$ \\
\hline & B & 69.7 & 0.6 & & $\mathrm{~V}$ & 119.4 & 2.6 \\
\hline & $\mathrm{V}$ & 70.0 & 0.4 & & $\mathrm{R}$ & 120.1 & 1.8 \\
\hline & $\mathrm{R}$ & 68.4 & 0.5 & & $\mathrm{I}$ & 120.0 & 7.7 \\
\hline & $\mathrm{I}$ & 68.5 & 1.5 & 7 & $\mathrm{U}$ & 68.9 & 4.4 \\
\hline & & MK 421 & & & $\mathrm{~B}$ & 68.2 & 3.2 \\
\hline \multirow[t]{5}{*}{1} & $\mathrm{U}$ & 10.7 & 2.4 & & $\mathrm{~V}$ & 69.4 & 2.8 \\
\hline & B & 12.6 & 2.7 & & $\mathrm{R}$ & 69.5 & 1.8 \\
\hline & $\mathrm{V}$ & 14.2 & 2.3 & & I & 70.1 & 3.8 \\
\hline & $\mathrm{R}$ & 4.4 & 2.1 & 8 & $\mathrm{U}$ & 69.1 & 2.5 \\
\hline & $\mathrm{I}$ & 9.4 & 6.0 & & $\mathrm{~B}$ & 70.3 & 3.6 \\
\hline \multirow[t]{6}{*}{2} & $\mathrm{U}$ & 10.5 & 4.0 & & $\mathrm{~V}$ & 72.4 & 2.1 \\
\hline & B & 9.3 & 2.9 & & $\mathrm{R}$ & 72.5 & 1.7 \\
\hline & $\mathrm{V}$ & 1.5 & 2.7 & & $\mathrm{I}$ & 73.7 & 3.3 \\
\hline & $\mathrm{R}$ & 0.6 & 1.3 & 9 & $\mathrm{U}$ & 63.1 & 2.2 \\
\hline & I & 172.6 & 2.6 & & B & 64.5 & 1.9 \\
\hline & & PKS 2155-304 & & & $\mathrm{V}$ & 66.7 & 1.6 \\
\hline \multirow[t]{5}{*}{1} & $\mathrm{U}$ & 93.1 & 3.9 & & $\mathrm{R}$ & 67.0 & 1.3 \\
\hline & B & 96.4 & 2.9 & & I & 67.7 & 2.5 \\
\hline & $\mathrm{V}$ & 96.7 & 2.7 & 10 & $\mathrm{U}$ & 113.8 & 7.3 \\
\hline & $\mathrm{R}$ & 97.1 & 2.7 & & B & 113.7 & 5.7 \\
\hline & I & 96.5 & 4.6 & & $\mathrm{~V}$ & 116.6 & 4.9 \\
\hline \multirow[t]{5}{*}{2} & $\mathrm{U}$ & 12.0 & 5.8 & & $\mathrm{R}$ & 115.9 & 4.7 \\
\hline & B & 4.3 & 7.4 & & I & 117.1 & 5.3 \\
\hline & V & 6.4 & 3.8 & 11 & $\mathrm{U}$ & 105.0 & 2.6 \\
\hline & $\mathrm{R}$ & 8.6 & 3.7 & & B & 105.7 & 2.5 \\
\hline & I & 8.3 & 11.3 & & V & 105.6 & 1.8 \\
\hline \multirow[t]{5}{*}{3} & $\mathrm{U}$ & 101.3 & 3.0 & & $\mathrm{R}$ & 106.1 & 1.3 \\
\hline & B & 104.1 & 3.9 & & I & 106.0 & 3.0 \\
\hline & $\mathrm{V}$ & 102.4 & 2.0 & 12 & $\mathrm{U}$ & 109.8 & 3.5 \\
\hline & $\mathrm{R}$ & 101.5 & 2.3 & & B & 110.4 & 4.3 \\
\hline & I & 101.0 & 3.4 & & V & 109.3 & 2.4 \\
\hline \multirow[t]{5}{*}{4} & $\mathrm{U}$ & 118.8 & 6.0 & & $\mathrm{R}$ & 108.1 & 2.1 \\
\hline & B & 121.6 & 3.1 & & I & 107.8 & 5.8 \\
\hline & V & 120.9 & 2.9 & 13 & $\mathrm{U}$ & 97.5 & 3.1 \\
\hline & $\mathrm{R}$ & 120.5 & 3.4 & & B & 88.3 & 4.8 \\
\hline & I & 121.1 & 5.9 & & $\mathrm{~V}$ & 96.4 & 2.8 \\
\hline \multirow[t]{5}{*}{5} & $\mathrm{U}$ & 125.4 & 4.0 & & $\mathrm{R}$ & 95.5 & 2.5 \\
\hline & B & 130.1 & 3.2 & & I & 99.3 & 4.3 \\
\hline & $\mathrm{V}$ & 131.5 & 1.6 & 14 & $\mathrm{U}$ & 91.8 & 3.8 \\
\hline & $\mathrm{R}$ & 131.3 & 3.0 & & B & 94.3 & 5.3 \\
\hline & $\mathrm{I}$ & 129.6 & 4.7 & & $\mathrm{~V}$ & 95.3 & 8.5 \\
\hline \multirow[t]{2}{*}{6} & $\mathrm{U}$ & 115.9 & 4.6 & & $\mathrm{R}$ & 95.6 & 5.6 \\
\hline & B & 119.1 & 3.3 & & I & 92.9 & 6.9 \\
\hline
\end{tabular}

We compile the polarization data from these bright blazars and get the wavelengths $\left(\lambda_{U_{\text {eff }}}=0.360 \mu \mathrm{m}, \lambda_{B_{\text {eff }}}=0.440 \mu \mathrm{m}, \lambda_{V_{\text {eff }}}=0.530 \mu \mathrm{m}, \lambda_{R_{\text {eff }}}=0.690 \mu \mathrm{m}\right.$, and $\left.\lambda_{I_{\text {eff }}}=0.830 \mu \mathrm{m}\right)$ as well as the corresponding polarization angles. If each group of spectropolarimetry data is separately used to constrain the parameter $\eta$, we would have slightly different results for the same blazar. Therefore, we allow a variation of the polarization from one time period to another and consider only the time-averaged polarization angles for each blazar. We take blazar 3C 66A as an example, which has eight groups of polarization data. For every wavelength bin, the time-averaged polarization angles for 3C 66 A during eight observational periods are computed through $\bar{\phi}_{\mathrm{obs}}=\sum_{i} \phi_{\mathrm{obs}, i} / 8$. The scattering in the shift between $\phi_{\mathrm{obs}, i}$ and $\bar{\phi}_{\mathrm{obs}}$, i.e., $\sigma_{\phi_{i}}=\left|\phi_{\mathrm{obs}, i}-\bar{\phi}_{\mathrm{obs}}\right|$, gives an estimate of the errors in $\bar{\phi}_{\mathrm{obs}}$, i.e., $\sigma_{\bar{\phi}}=\left(\sum_{i} \sigma_{\phi_{i}}^{2}\right)^{1 / 2} / 8$.

We now have five groups of polarization data for the selected five blazars compiled with the time-averaged polarization angles and their corresponding energies. For the linear regression applied to the multiwavelength polarization observations of each blazar, we use the function $\phi_{\text {obs }}$ (Equation (6)) to fit the observed data. The Metropolis-Hastings Markov chain Monte Carlo (MHMC) method is applied to simulate the free parameters $\phi_{0}$ and $B$ of the linear function. The best-fitting results of multiwavelength polarization observations for five blazars are presented in Table 2. For each blazar in turn, the best- 
fitting values and $2 \sigma$ uncertainties are provided for the parameters $\phi_{0}$ and $B$ and the corresponding birefringence parameter $\eta$. The time-averaged polarization angles of the five blazars as a function of $E^{2}$ are shown in Figure 1, and the red lines are the best-fitting lines. As shown in Figure 1, the polarization angles of blazars S5 0716+714 and PKS 2155-304 are proportional to $E^{2}$ with a negative coefficient, and the best-fitting values are $\eta=(-9.63 \pm 4.640) \times 10^{-8}\left(\phi_{0}=0.320 \pm 0.058 \mathrm{rad}\right)$ and $\eta=(-5.32 \pm 3.587) \times 10^{-7}$ $\left(\phi_{0}=1.645 \pm 0.018 \mathrm{rad}\right)$, respectively. While the rest of the blazars show a positive coefficient with the fitting parameters $\eta=(3.386 \pm 1.249) \times 10^{-7}\left(\phi_{0}=0.623 \pm 0.024 \mathrm{rad}\right)$, $\eta=(6.546 \pm 22.769) \times 10^{-6}\left(\phi_{0}=0.046 \pm 0.482 \mathrm{rad}\right)$, and $\eta=(6.226 \pm 7.715) \times 10^{-7}$ $\left(\phi_{0}=1.544 \pm 0.094 \mathrm{rad}\right)$ for 3C 66A, MK 421, and OJ 287, respectively. Our constraints show that except for 3C 66A at the 5.4 $\sigma$ confidence level and PKS 2155-304 at the 3.0 confidence level, the data set of the other three blazars are consistent with the possibility of no LIV at all (i.e., $\eta=0$ ) within the $1.8 \sigma$ confidence level. The wavelength dependence of polarization angles, even if present in 3C 66A and PKS 2155-304, cannot be due to LIV effects as this would clash with the best limit from S5 $0716+714$ by factors of 4 to 6 , and must therefore be of intrinsic astrophysical origin. At the $2 \sigma$ confidence level, the limits on $\eta$ from five blazars are $-9.63 \times 10^{-8}<\eta<6.55 \times 10^{-6}$.

Table 2. Best-fitting results of multiwavelength polarization observations for five blazars.

\begin{tabular}{ccccc}
\hline Source & $\boldsymbol{z}$ & $\mathbf{B}$ & $\boldsymbol{\phi}_{\mathbf{0}}$ (rad) & $\left.\boldsymbol{\eta}_{(\times \mathbf{1 0}}^{\mathbf{- 7}}\right)$ \\
\hline 3C 66A & 0.444 & $0.009 \pm 0.003$ & $0.623 \pm 0.024$ & $3.386 \pm 1.249$ \\
S5 0716+714 & 0.310 & $-0.002 \pm 0.009$ & $0.320 \pm 0.058$ & $-0.963 \pm 4.640$ \\
OJ 287 & 0.306 & $0.012 \pm 0.013$ & $1.544 \pm 0.094$ & $6.226 \pm 7.175$ \\
MK 421 & 0.031 & $0.012 \pm 0.041$ & $0.046 \pm 0.482$ & $65.464 \pm 227.692$ \\
PKS 2155-304 & 0.116 & $-0.004 \pm 0.002$ & $1.645 \pm 0.018$ & $-5.322 \pm 3.587$ \\
\hline
\end{tabular}
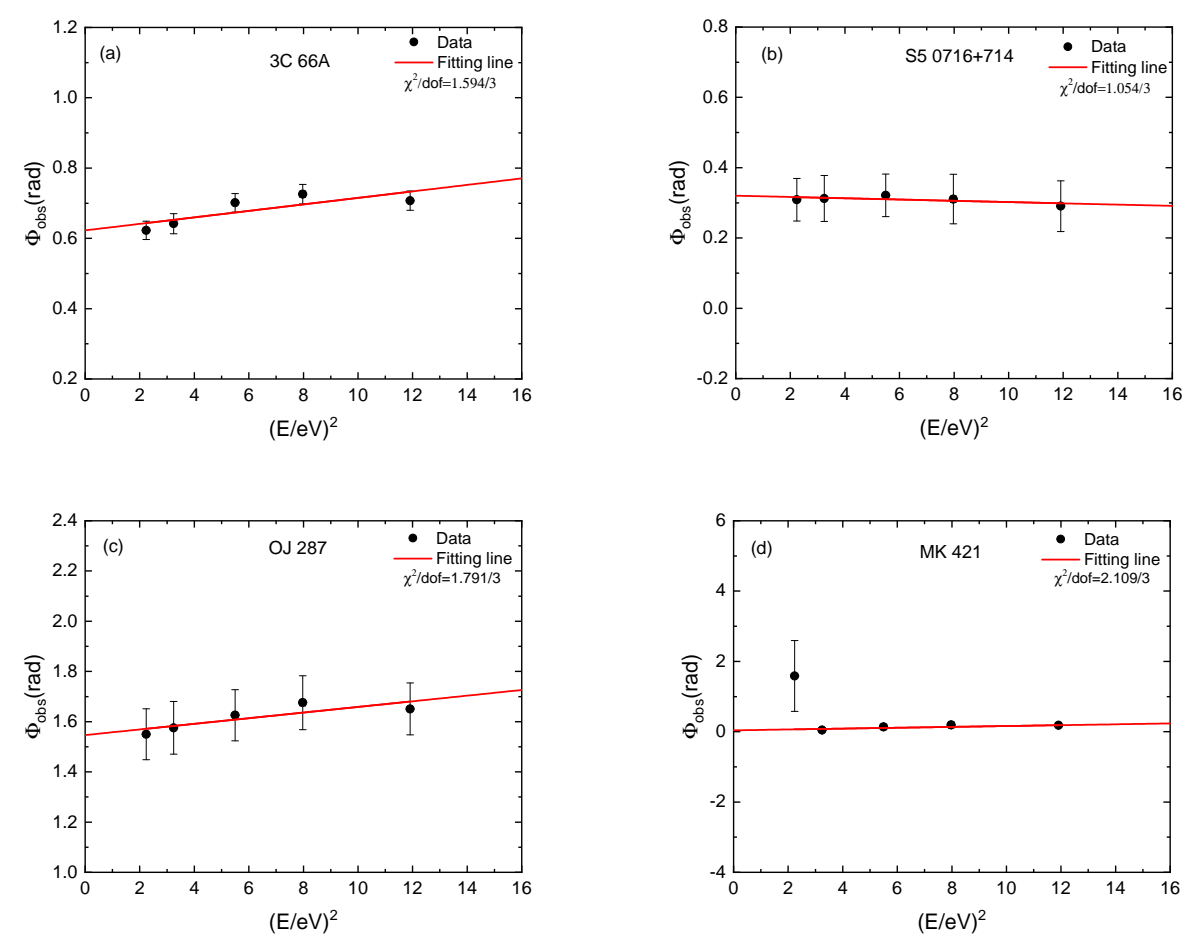

Figure 1. Cont. 


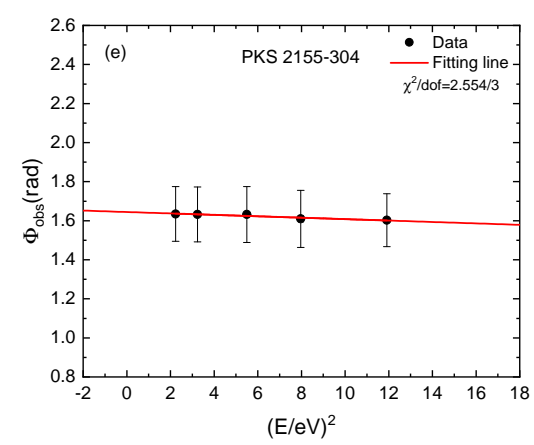

Figure 1. Best -fitting results of the optical spectropolarimetric data of five blazars. (a): Linear fit of 3C 66A. (b): Linear fit of S5 0716+714. (c): Linear fit of OJ 287. (d): Linear fit of MK 421. (e): Linear fit of PKS 2155-304. The black dots correspond to the time-averaged linear polarization data, and the red lines are the theoretical curves based on Equation (6).

\section{Discussion}

According to the summary of the limits on the birefringence parameter $\eta$ from the polarization measurements of various astrophysical sources [54], we can simply make a comparison with our results. The polarization observations on the prompt $\gamma$-ray emission of GRBs place the hitherto most stringent constraints on $\eta$, i.e., $\eta<\mathcal{O}\left(10^{-16}\right)$ [29-33]. As expected, our optical polarization constraints are not competitive with that from the observations of gamma-ray polarization. However, these gamma-ray polarization limits suffer from a relatively low statistical confidence of the polarization measurements and large systematic uncertainties. In the optical band, as we do, Gleiser \& Kozameh [48] set an upper limit of $\eta<10^{-4}$ by processing the linearly polarized ultraviolet light from the radio galaxy 3C 256, and Fan et al. [39] obtained the limits on $\eta$ of $-2 \times 10^{-7}<\eta<1.4 \times 10^{-7}$ by using the ultraviolet/optical polarization data of the afterglows of GRBs. We have used the optical polarimetric observations in five bands (UBVRI) of blazars to derive the limits $-9.63 \times 10^{-8}<\eta<6.55 \times 10^{-6}$ at the $2 \sigma$ confidence level, which is essentially as good as or one order of magnitude improvement over previous limits obtained by ultraviolet/optical polarization observations.

It is worth pointing out that Kislat \& Krawczynski [34] also used the optical polarization measurements to constrain LIV. They introduced Lorentz and CPT symmetry-violating effects by adding new terms which can be ordered by the mass dimension of the corresponding field operator in the Standard Model Lagrangian. They analyzed optical polarization data from 72 AGNs and GRBs and obtained a set of constraints on the 16 coefficients of mass dimension $d=5$ of the Standard Model Extension photon sector. Their results implied a lower limit on the energy scale of quantum gravity of $10^{5}$ times the Planck energy. The approach in [34] constrained LIV by limiting the coefficients of $d=5$, while we got the results by constraining the birefringence parameter $\eta$. Both approaches are rigorous and constrain LIV well.

Note that we have used the standard flat $\Lambda \mathrm{CDM}$ model for our calculations (see Equation (4)). However, other different cosmological models have been proposed. In these models, dark energy evolves in a way that is different from that in the $\Lambda$ CDM model. Therefore, it is necessary to study whether LIV is caused by the particular model of the universe we are applying. Moreover, apart from dark energy, the contribution of spatial curvature should not be ignored. Refs. $[55,56]$ have investigated these issues in different cosmological models. By extending the analysis performed in the $\Lambda \mathrm{CDM}$ model to other cosmological models, they found that the effects, e.g, the redshift dependence of time delays caused by LIV, also occurs in alternative cosmological models.

It should be stressed that we have performed the analysis based on the assumption of isotropy. On the other hand, anisotropic LIV has been discussed in theory, and con- 
straints on anisotropic LIV have also been obtained from some relevant observations, see, e.g., Refs. [12,14,34].

\section{Conclusions}

As a consequence of LIV, the group velocities of left- and right-handed circularly polarized photons that are emitted from the same astrophysical source should differ slightly, leading to vacuum birefringence and a frequency-dependent rotation of the polarization vector of linearly polarized light. LIV can therefore be tested with linear polarization measurements of astrophysical sources. A key challenge in the idea of searching for a frequency-dependent change in polarization, however, is to distinguish the rotation angle induced by LIV from any source-intrinsic polarization angle in the emission of photons in different energy bands. Following the method of Fan et al. [39], we simply assume that the intrinsic polarization angle is an unknown constant and expect to observe birefringence effects as a frequency-dependent linear polarization vector.

To constrain LIV, we try to search for a similar frequency-dependent trend in multiwavelength polarization observations of astrophysical sources. In this work, five blazars with multiwavelength polarization angles in five optical bands (UBVRI) are collected. For each blazar, the optical polarization measurements were carried out at different time periods and, thus, there are a total of 37 groups of spectropolarimetry data for the five blazars. Considering the temporal variation of the polarization observations, we extract the time-averaged polarization angles in five wavelength bins for each selected blazar. We then use the function $\phi_{\text {obs }}(E)$ (Equation (6)) to fit the time-averaged polarization angles versus the quantities of $E^{2}$, and obtain the best-fitting results of the intrinsic polarization angle $\phi_{0}$ and the birefringence parameter $\eta$. At the $2 \sigma$ confidence level, we find that $\eta$ is constrained to be in the range of $-9.63 \times 10^{-8}<\eta<6.55 \times 10^{-6}$ for five blazars. These results are comparable with or represent sensitivities improved by one order of magnitude over existing limits from ultraviolet/optical polarization observations. More stringent limits on the birefringence parameter can be expected as the analysis presented here is applied to a larger number of astrophysical sources with larger distances and higher energy polarimetry.

Note that the rotation of the linear polarization plane can also be caused by magnetized plasmas, where this phenomenon is known as Faraday rotation. Hence, it is necessary to investigate whether our resulting constraints could be affected by Faraday rotation. The dependence of the rotation angle on Faraday rotation is $\Delta \Phi_{\mathrm{Far}} \propto E^{-2}$, different from its dependence on LIV effects as $\Delta \Phi_{\text {LIV }} \propto E^{2}$ shown in Equation (5). According to the discussions of Fan et al. [39] and Wei \& Wu [46], the rotation angle $\Delta \Phi_{\mathrm{Far}}$ is extremely small at the optical and higher energy band. Therefore, Faraday rotation can be ignored in our analysis.

Author Contributions: Q.-Q.Z. and S.-X.Y. analyzed the observational data and explained the fitting results of multiwavelength polarization measurements in different optical bands from five blazars. J.-J.W. and X.-F.W. suggested that LIV can be tested with linear polarization measurements of astrophysical sources. All of the authors wrote this paper together. All authors have read and agreed to the published version of the manuscript.

Funding: This research was funded by the National Natural Science Foundation of China (Grant Nos. 11703015, U2038106, 11725314, U1831122, and 12041306), the Youth Innovations and Talents Project of Shandong Provincial Colleges and Universities (Grant No. 201909118), the Youth Innovation Promotion Association (2017366), the Key Research Program of Frontier Sciences (grant No. ZDBSLY-7014), the Strategic Priority Research Program "Multi-waveband gravitational wave universe" (grant No. XDB23000000) of the Chinese Academy of Sciences, and the Major Science and Technology Project of Qinghai Province (2019-ZJ-A10).

Institutional Review Board Statement: Not applicable.

Informed Consent Statement: Not applicable.

Data Availability Statement: The data underlying this article are available in the article. 
Acknowledgments: The authors would like to thank the referees for their comments, which significantly helped us improving the presentation of the paper. We thank Yu-Peng Yang for helpful discussions.

Conflicts of Interest: The authors declare no conflicts of interest.

\section{References}

1. Tasson, J.D. What do we know about Lorentz invariance? Rep. Prog. Phys. 2014, 77, 062901. [CrossRef]

2. Amelino-Camelia, G. Quantum-Spacetime Phenomenology. Living Rev. Relativ. 2013, 16, 5. [CrossRef]

3. Mattingly, D. Modern Tests of Lorentz Invariance. Living Rev. Relativ. 2005, 8, 5. [CrossRef]

4. Mitrofanov, I.G. Astrophysics (communication arising): A constraint on canonical quantum gravity? Nature 2003, 426, 139. [CrossRef]

5. Kostelecký, V.A.; Potting, R. CPT, strings, and meson factories. Phys. Rev. D 1995, 51, 3923. [CrossRef]

6. Kostelecký, V.A.; Potting, R. CPT and strings. Nucl. Phys. B 1991, 359, 545. [CrossRef]

7. Kostelecký, V.A.; Samuel, S. Spontaneous breaking of Lorentz symmetry in string theory. Phys. Rev. D 1989, 39, 683. [CrossRef]

8. Amelino-Camelia, G.; Ellis, J.; Mavromatos, N.E.; Nanopoulos, D.V.; Sarkar, S. Tests of quantum gravity from observations of $\gamma$-ray bursts. Nature 1998, 393, 763. [CrossRef]

9. Acciari, V.A.; Ansoldi, S.; Antonelli, L.A.; Arbet Engels, A.; Baack, D.; Babić, A.; Banerjee, B.; Barres de Almeida, U.; Barrio, J.A.; Becerra Gonz ález, J. et al. Bounds on Lorentz Invariance Violation from MAGIC Observation of GRB 190114C. Phys. Rev. Lett. 2020, 125, 021301. [CrossRef]

10. Ellis, J.; Konoplich, R.; Mavromatos, N.E.; Nguyen, L.; Sakharov, A.S.; Sarkisyan-Grinbaum, E.K. Robust constraint on Lorentz violation using Fermi-LAT gamma-ray burst data. Phys. Rev. D 2019, 99, 083009. [CrossRef]

11. Wei, J.-J.; Wu, X.-F. A Further Test of Lorentz Violation from the Rest-frame Spectral Lags of Gamma-Ray Bursts. Astrophys. J. 2017, 851, 127. [CrossRef]

12. Wei, J.-J.; Wu, X.-F.; Zhang, B.-B.; Shao, L.; Mészáros, P.; Kostelecký, V.A. Constraining Anisotropic Lorentz Violation via the Spectral-lag Transition of GRB 160625B. Astrophys. J. 2017, 842, 115. [CrossRef]

13. Wei, J.-J.; Zhang, B.-B.; Shao, L.; Wu, X.-F.; Mészáros, P. A New Test of Lorentz Invariance Violation: The Spectral Lag Transition of GRB 160625B. Astrophys. J. 2017, 834, L13. [CrossRef]

14. Kislat, F.; Krawczynski, H. Search for anisotropic Lorentz invariance violation with $\gamma$-rays. Phys. Rev. D 2015, 92, 045016. [CrossRef]

15. Zhang, S.; Ma, B.-Q. Lorentz violation from gamma-ray bursts. Astropart. Phys. 2015, 61, 108. [CrossRef]

16. Ellis, J.; Mavromatos, N.E. Probes of Lorentz violation. Astropart. Phys. 2013, 43, 50. [CrossRef]

17. Vasileiou, V.; Jacholkowska, A.; Piron, F.; Bolmont, J.; Couturier, C.; Granot, J.; Stecker, F.W.; Cohen-Tanugi, J.; Longo, F. Constraints on Lorentz invariance violation from Fermi-Large Area Telescope observations of gamma-ray bursts. Phys. Rev. D 2013, 87, 122001. [CrossRef]

18. Chang, Z.; Jiang, Y.; Lin, H.-N. A unified constraint on the Lorentz invariance violation from both short and long GRBs. Astropart. Phys. 2012, 36, 47. [CrossRef]

19. Nemiroff, R.J.; Connolly, R.; Holmes, J.; Kostinski, A.B. Bounds on Spectral Dispersion from Fermi-Detected Gamma Ray Bursts. Phys. Rev. Lett. 2012, 108, 231103. [CrossRef]

20. Abdo, A.A.; Ackermann, M.; Ajello, M.; Asano, K.; Atwood, W.B.; Axelsson, M.; Baldini, L.; Ballet, J.; Barbiellini, G. A limit on the variation of the speed of light arising from quantum gravity effects. Nature 2009, 462, 331. [CrossRef]

21. Kostelecký, V.A.; Mewes, M. Electrodynamics with Lorentz-violating operators of arbitrary dimension. Phys. Rev. D 2009, 80, 015020. [CrossRef]

22. Abdo, A.A.; Ackermann, M.; Arimoto, M.; Asano, K.; Atwood, W.B.; Axelsson, M.; Baldini, L.; Ballet, J.; Band, D.L. Fermi Observations of High-Energy Gamma-Ray Emission from GRB 080916C. Science 2009, 323, 1688. [PubMed]

23. Jacob, U.; Piran, T. Lorentz-violation-induced arrival delays of cosmological particles. J. Cosmol. Astropart. Phys. $2008,2008,031$. [CrossRef]

24. Ellis, J.; Mavromatos, N.E.; Nanopoulos, D.V.; Sakharov, A.S.; Sarkisyan, E.K.G. Robust limits on Lorentz violation from gamma-ray bursts. Astropart. Phys. 2006, 25, 402. [CrossRef]

25. Pavlopoulos, T.G. Are we observing Lorentz violation in gamma ray bursts? [rapid communication]. Phys. Lett. B 2005, 625, 13. [CrossRef]

26. Kostelecký, V.A.; Mewes, M. Astrophysical Tests of Lorentz and CPT Violation with Photons. Astrophys. J. 2008, 689, L1. [CrossRef]

27. Kaaret, P. Pulsar radiation and quantum gravity. Astron. Astrophys. 1999, 345, L32.

28. Biller, S.D.; Breslin, A.C.; Buckley, J.; Catanese, M.; Carson, M.; Carter-Lewis, D.A.; Cawley, M.F.; Fegan, D.J.; Finley, J. P. Limits to Quantum Gravity Effects on Energy Dependence of the Speed of Light from Observations of TeV Flares in Active Galaxies. Phys. Rev. Lett. 1999, 83, 2108. [CrossRef]

29. Wei, J.-J. New constraints on Lorentz invariance violation with polarized gamma-ray bursts. Mon. Not. R. Astron. Soc. 2019, 485, 2401. [CrossRef]

30. Lin, H.-N.; Li, X.; Chang, Z. Gamma-ray burst polarization reduction induced by the Lorentz invariance violation. Mon. Not. R. Astron. Soc. 2016, 463, 375. [CrossRef] 
31. Götz, D.; Laurent, P.; Antier, S.; Covino, S.; D'Avanzo, P.; D’Elia, V.; Melandri, A. GRB 140206A: The most distant polarized gamma-ray burst. Mon. Not. R. Astron. Soc. 2014, 444, 2776. [CrossRef]

32. Götz, D.; Covino, S.; Fernández-Soto, A.; Laurent, P.; Bošnjak, Ž. The polarized gamma-ray burst GRB 061122. Mon. Not. R. Astron. Soc. 2013, 431, 3550. [CrossRef]

33. Toma, K.; Mukohyama, S.; Yonetoku, D.; Murakami, T.; Gunji, S.; Mihara, T.; Morihara, Y.; Sakashita, T.; Takahashi, T. Strict Limit on CPT Violation from Polarization of $\gamma$-Ray Bursts. Phys. Rev. Lett. 2012, 109, 241104. [CrossRef]

34. Kislat, F.; Krawczynski, H. Planck-scale constraints on anisotropic Lorentz and C P T invariance violations from optical polarization measurements. Phys. Rev. D 2017, 95, 083013. [CrossRef]

35. Friedman, A.S.; Leon, D.; Crowley, K.D.; Johnson, D.; Teply, G.; Tytler, D.; Keating, B.G.; Cole, G.M. Constraints on Lorentz invariance and C P T violation using optical photometry and polarimetry of active galaxies BL Lacertae and S5 B $0716+714$. Phys. Rev. D 2019, 99, 035045 [CrossRef]

36. Stecker, F.W. A new limit on Planck scale Lorentz violation from $\gamma$-ray burst polarization. Astropart. Phys. 2011, 35, 95. [CrossRef]

37. Laurent, P.; Götz, D.; Binétruy, P.; Covino, S.; Fernandez-Soto, A. Constraints on Lorentz Invariance Violation using integral/IBIS observations of GRB041219A. Phys. Rev. D 2011, 83, 121301. [CrossRef]

38. Gubitosi, G.; Pagano, L.; Amelino-Camelia, G.; Melchiorri, A.; Cooray, A. A constraint on Planck-scale modifications to electrodynamics with CMB polarization data. J. Cosmol. Astropart. Phys. 2009, 2009, 021. [CrossRef]

39. Fan, Y.-Z.; Wei, D.-M.; Xu, D. $\gamma$-ray burst ultraviolet/optical afterglow polarimetry as a probe of quantum gravity. Mon. Not. $R$. Astron. Soc. 2007, 376, 1857. [CrossRef]

40. Kostelecký, V.A.; Mewes, M. Constraints on Relativity Violations from Gamma-Ray Bursts. Phys. Rev. Lett. 2013, $110,201601$. [CrossRef]

41. Kostelecký, V.A.; Mewes, M. Lorentz-Violating Electrodynamics and the Cosmic Microwave Background. Phys. Rev. Lett. 2007, 99, 011601. [CrossRef]

42. Kostelecký, V.A.; Mewes, M. Sensitive Polarimetric Search for Relativity Violations in Gamma-Ray Bursts. Phys. Rev. Lett. 2006, 97, 140401. [CrossRef]

43. Kostelecký, V.A.; Mewes, M. Cosmological Constraints on Lorentz Violation in Electrodynamics. Phys. Rev. Lett. 2001, 87, 251304. [CrossRef]

44. Colladay, D.; Kostelecký, V.A. Lorentz-violating extension of the standard model. Phys. Rev. D 1998, 58, 116002. [CrossRef]

45. Carroll, S.M.; Field, G.B.; Jackiw, R. Limits on a Lorentz- and parity-violating modification of electrodynamics. Phys. Rev. D 1990, 41, 1231. [CrossRef] [PubMed]

46. Wei, J.-J.; Wu, X.-F. Testing the weak equivalence principle and Lorentz invariance with multiwavelength polarization observations of GRB optical afterglows. Eur. Phys. J. Plus 2020, 135, 527. [CrossRef]

47. Jacobson, T.; Liberati, S.; Mattingly, D.; Stecker., F.W. New Limits on Planck Scale Lorentz Violation in QED. Phys. Rev. Lett. 2004, 93, 021101. [CrossRef]

48. Gleiser, R.J.; Kozameh, C.N. Astrophysical limits on quantum gravity motivated birefringence. Phys. Rev. D 2001, 64, 083007. [CrossRef]

49. Gambini, R.; Pullin, J. Nonstandard optics from quantum space-time. Phys. Rev. D 1999, 59, 124021. [CrossRef]

50. Aghanim, N.; Akrami, Y.; Ashdown, M.; Aumont, J.; Baccigalupi, C.; Ballardini, M.; Banday, A.J.; Barreiro, R.B.; Bartolo, N.; Basak, S.; et al. Planck Collaboration. Planck 2018 results. VI. Cosmological parameters. Astron. Astrophys. 2020, 641, A6.

51. Tommasi, L.; Díaz, R.; Palazzi, E.; Pian, E.; Poretti, E.; Scaltriti, F.; Treves, A. Multiband Optical Polarimetry of the BL Lacertae Object PKS 2155-304: Intranight and Long-Term Variability. Astrophys. J. Suppl. Ser. 2001, 132, 73. [CrossRef]

52. Takalo, L.O.; Sillanpaeae, A.; Nilsson, K. Simultaneous UBVRI photopolarimetry of three blazars: 3C 66A, S5 0716+714 and OJ 287. Astron. Astrophys. Suppl. Ser. 1994, 107, 497.

53. Takalo, L.O.; Sillanpaa, A. Simultaneous linear and circular polarization observations of blazars 3C 66A, OJ 287 and Markarian 421. Astrophys. Space Sci. 1993, 206, 191. [CrossRef]

54. Wei, J.-J.; Wu, X.-F. Testing fundamental physics with astrophysical transients. Front. Phys. 2021, 16, 44300. [CrossRef]

55. Biesiada, M.; Piórkowska, A. Lorentz invariance violation-induced time delays in GRBs in different cosmological models. Class. Quantum Grav. 2009, 26, 125007. [CrossRef]

56. Luongo, O.; Tommasini, D. Cosmological Measurements of Lorentz Invariance Violation at the LIFŠHITZ Point. Int. J. Mod. Phys. D 2012, 21, 1250070. [CrossRef] 